\title{
Expanding atmospheres in non-LTE:
}

\section{Radiation transfer using short characteristics}

\author{
L. Koesterke, W.-R. Hamann, and G. Gräfener \\ Professur Astrophysik, Universität Potsdam, Am Neuen Palais 10, 14469 Potsdam, Germany
}

Received 14 March 2001 / Accepted 10 October 2001

\begin{abstract}
We present our technique for solving the equations of radiation transfer in spherically expanding atmospheres. To ensure an efficient treatment of the Thomson scattering, the mean intensity $J$ is derived by solving the moment equations in turn with the angle-dependent transfer equation. The latter provide the Eddington factors. Two different methods for the solution of the angle dependent equation are compared. Thereby the integration along short characteristics turned out to be superior in our context over the classical differencing scheme. The method is the basis of a non-LTE code suitable for the atmospheres of hot stars with high mass-loss.
\end{abstract}

Key words. radiative transfer - methods: numerical - stars: atmospheres

\section{Introduction}

The development of the Accelerated Lambda Iteration (ALI) method in the last fifteen years has stimulated great progress in modelling non-LTE atmospheres of hot stars. Nowadays it is possible to account for hundreds of levels, thousands of lines and, due to the introduction of the super level concept (Anderson 1989), for the opacity of millions of iron lines in non-LTE. Plane-parallel models of this type, adequate for the static atmospheres of hot and compact objects, have been developed by Werner and coworkers (e.g. Dreizler \& Werner 1993) and by Hubeny and co-workers (e.g. Hubeny \& Lanz 1995) in the mid-nineties, while corresponding models for spherically expanding atmospheres were developed few years later (Hillier \& Miller 1998; HM in the following). A comprehensive overview of the various types of non-LTE codes for stellar atmospheres is given in HM.

The fundamental difference between static and expanding models is the differential motion of the plasma in the expanding atmospheres. A special numerical treatment is required:

- spherical symmetry has to be accounted for because the size of the atmospheres is comparable to the size of the star. As a consequence the number of angles is comparable to the number of depth points;

- to consider the Doppler shift of the differential moving atmosphere a co-moving system of reference (CMF) is favorable;

Send offprint requests to: L. Koesterke, e-mail: lars@astro.physik.uni-potsdam.de
- due to the differential Doppler shift the frequencies are coupled. To keep the problem manageable a monotonically increasing velocity law is indispensable. Then the radiation field at a given frequency depends only on local quantities and the radiation field at the neighboring blue frequency.

The goal is to calculate radiation fields at all frequencies and depths consistent with non-LTE population numbers of all levels at all depths. Obviously the solution as a whole, with ten of thousands of frequencies, hundreds of levels and dozens of depth points, is extremely difficult because the number of variables easily exceeds $10^{9}$ for complex models.

The basic idea of the ALI scheme is to split the calculation into two parts. In the first step the radiation field is calculated from the non-LTE population numbers which are kept fixed meanwhile. In the second step new population numbers are calculated from the radiation fields by solving the equations of statistical equilibrium. In order to achieve a consistent solution both steps are repeated. To overcome problems in optically thick situations the iteration is accelerated by additional non-linear terms in the equations of statistical equilibrium.

In the present paper we describe our implementation of the radiation transfer for spherically expanding atmospheres in the co-moving frame (i.e. step one in the ALI scheme). The solution of the equations of the statistical equilibrium will be the subject of a subsequent paper (Koesterke in prep.). Our approach is similar to that presented by HM. Therefore we do not repeat all computational details but concentrate on the differences which 
mainly concern the calculation of the angle-dependent intensity $I$. After an overview on the concept (Sect. 2) and the basic equations (Sect.3) we report on the solution of the ray-by-ray radiation transfer applying a differencing and a short characteristic scheme, respectively (Sect.4). A summary is given in Sect. 5 .

\section{Overview}

In contrast to our previous non-LTE models (Hamann 1987) continua and lines are not calculated separately. All opacities and emissivities at a given frequency are added and the radiation transfer equation is solved. This allows us to treat "exactly" millions of iron lines which are incorporated via the super level concept (Anderson 1989) without any opacity distribution or sampling technique. A detailed description of our implementation of the super level concept will be given in Gräfener et al. (in prep.).

The straight method to evaluate the radiation field at a given frequency from a given source function (i.e. fixed population numbers) is the calculation along each ray (in the following: ray-by-ray calculation). To account for the angle coupling due to Thomson scattering by free electrons, either all angles have to be considered at once or the calculation has to be iterated. The former method, the consideration of all angles at once, leads to a tridiagonal block matrix of rank ND (ND: number of depths) with all matrix elements being block matrices of rank NA (NA: number of angles) themselves. In spherically extended models with $\mathrm{NA} \gtrsim \mathrm{ND} \approx 50$ this would be a very time consuming calculation. The latter method is also slow due to its internal iterations.

Therefore we decided to follow HM and apply the time-saving method of variable Eddington factors (Auer \& Mihalas 1970). Within this method the radiation field is calculated from the moment equations. The Thomson scattering is explicitely allowed for under the assumption of coherence. For the closure of the set of moment equations, Eddington factors are calculated in a ray-by-ray scheme typically every sixth ALI cycle. The very first ALI cycle starts with the ray-by-ray scheme applying continuum radiation fields from a static approximation.

To keep the number of frequency points small we introduce a microturbulence $v_{\text {Dop }}=100 \mathrm{~km} \mathrm{~s}^{-1}$. No other broadening mechanism is accounted for, so far. In the vicinity of lines and continuum edges (i.e. from $-4.5 v_{\text {Dop }}$ up to $\left.4.5 v_{\mathrm{D}}+2 v_{\infty}\right)$ the frequency spacing is set to $0.3 v_{\text {Dop }}$. For the broad lines between super levels the range is analogously increased. In all other spectral regions about 500 frequency points per decade are placed. Note that in realistic models with thousands of lines almost the whole spectral range is covered by the fine frequency grid. The velocity $v(r)$ is prescribed by the usual $\beta$-law. Typically about 50 depth points and 54 angles are taken for the spatial grid. It covers the range from the outer boundary at about $1000 R_{\odot}$ down to Rosseland optical depths of about 20. The electron scattering is calculated in coherent approximation.

\section{Equations of radiation transfer}

We apply the usual spherical coordinate system (Mihalas 1978 ) with the radial coordinate $r$, the impact parameter $p$, the coordinate $z$ on a ray and $\mu$ being the cosine between the direction of a ray and the radial direction. $v(r)$ denotes the monotonically increasing velocity of the atmosphere. The transfer equation in the CMF for an intensity $I$ at a given frequency $\nu$ is then given by

$$
\begin{aligned}
\mu \frac{\partial}{\partial r} I_{\nu, \mu, r} & +\frac{1-\mu^{2}}{r} \frac{\partial}{\partial \mu} I_{\nu, \mu, r} \\
& -\frac{\nu v(r)}{c r}\left[1-\mu^{2}+\mu^{2}\left(\frac{\mathrm{d} \ln v(r)}{\mathrm{d} \ln r}\right)\right] \frac{\partial I}{\partial \nu} I_{\nu, \mu, r} \\
= & \eta_{\nu, \mu, r}-\kappa_{\nu, \mu, r} I_{\nu, \mu, r}
\end{aligned}
$$

$\eta$ and $\kappa$ denote the emissivity and opacity, respectively. Because the velocities of the atmospheres under consideration are low compared to the speed of light $(v / c \lesssim 0.01)$ aberration and advection terms are neglected (Mihalas et al. 1976). Introducing the moments of the intensity

$[J, H, K, N]=\frac{1}{2} \int_{-1}^{1} I(\mu)\left[1, \mu, \mu^{2}, \mu^{3}\right] \mathrm{d} \mu$,

the Eddington factors

$f_{\nu, r}=\frac{K_{\nu, r}}{J_{\nu, r}}, \quad g_{\nu, r}=\frac{N_{\nu, r}}{H_{\nu, r}}$,

the sphericity factor $q$

$\ln \left(r^{2} q\right)=\int_{R_{*}}^{r}\left(\frac{3-1 / f}{r}\right) \mathrm{d} r+\ln R_{*}^{2}$,

and

$\mathrm{d} X=-\kappa q \mathrm{~d} r, \quad \alpha=\frac{\nu v}{\kappa c r}, \quad \beta=\frac{\mathrm{d} \ln v}{\mathrm{~d} \ln r}$

the zeroth and the first moment of the transfer equation can be written as

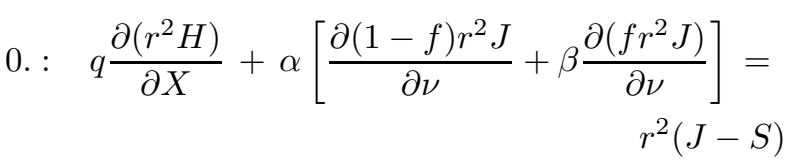

1. : $\frac{\partial\left(f q r^{2} J\right)}{\partial X}+\alpha\left[\frac{\partial(1-g) r^{2} H}{\partial \nu}+\beta \frac{\partial\left(g r^{2} H\right)}{\partial \nu}\right]=$

Utilizing the given Eddington factors $f$ and $g$ and special factors for the boundary, Eqs. (2) and (3) can be solved for the unknowns $J$ and $H$. This leads to a tridiagonal Feautrier scheme. The Eddington factors are calculated from the solution of the equation for the angle dependent intensity $I$ (Eq. (1)).

Similar to HM we replace in Eqs. (2) and (3) $g H=$ $N=g^{\prime}(H+\epsilon J) . \epsilon$ is chosen in a way that the term in brackets is always a positive number. Therewith $g^{\prime}$ is always a well-defined quantity. 
To provide the boundary in frequency, the Eqs. (1), (2) and (3) are solved for the bluemost frequency, neglecting the frequency derivatives. At the inner spatial boundary the diffusion approximation is adopted.

At the outer boundary we have to deal with many frequencies (typically below the He II edge and at resonance lines) at which the model is still optically thick. Due to the moderate density decrease with radius ( $\rho$ roughly scales with $1 / r^{2}$ ) one cannot extend the model to a radius where all frequencies are optically thin and where generally $I^{-}$could be set to zero. The outer boundary would then be shifted to more than $10^{6}$ times of $R_{*}$ in some cases. Instead, we derive the incident radiation $I^{-}$under simplifying assumptions for the opacities and source functions. This effort is justified because an improperly chosen incident radiation field at optically thick frequencies produces highly disturbing artefacts at neighboring optically thin frequencies. Note that these artefacts are not limited to the outer boundary but affect the whole outer region down to $\tau_{\nu} \approx 1$.

Under the assumption that opacities, source functions and densities dilute quadratically with the radius, the incident radiation field $I^{-}$is calculated. The small velocity gradient is neglected here which is justified because the outer boundary is located at a large radius. The optical depth is then defined by

$\tau(r)=\int_{r>r_{\mathrm{b}}}^{\infty} \kappa_{\mathrm{b}} \frac{r_{\mathrm{b}}^{2}}{r^{\prime 2}} \mathrm{~d} r^{\prime}=\frac{\kappa_{\mathrm{b}} r_{\mathrm{b}}^{2}}{r}$

with quantities at the outer boundary being indicated by b. With $\tau_{\mathrm{b}}=\tau\left(r_{\mathrm{b}}\right)$ the source function $S$ can be written as

$S(r)=S_{\mathrm{b}} \frac{r_{\mathrm{b}}^{2}}{r^{2}}=S_{\mathrm{b}} \frac{\tau(r)^{2}}{\tau_{\mathrm{b}}^{2}}$.

This leads to

$$
\begin{gathered}
I^{-}\left(r_{\mathrm{b}}\right)=\int_{0}^{\tau_{\mathrm{b}}} S_{\mathrm{b}} \frac{\tau^{2}}{\tau_{\mathrm{b}}^{2}} e^{\tau-\tau_{\mathrm{b}}} \mathrm{d} \tau= \\
S_{\mathrm{b}}\left(1-\frac{2}{\tau_{\mathrm{b}}}+\frac{2}{\tau_{\mathrm{b}}^{2}}-\frac{2 e^{-\tau_{\mathrm{b}}}}{\tau_{\mathrm{b}}^{2}}\right) .
\end{gathered}
$$

Best results have been achieved by not taking the source function at the boundary itself, but by fitting a polynomial of low order to the source function at the outermost radius points.

\section{Solution of the ray-by-ray transfer equation}

In order to solve Eq. (1) on a ray we introduce the dimensionless frequency scale

$x=\frac{v_{\text {Dop }}}{c} \ln \frac{\nu}{\nu_{\text {ref }}}$

with $v_{\text {Dop }}$ being the Doppler shift due to microturbulence and $\nu_{\text {ref }}$ being an arbitrary reference frequency. The transfer equation along a ray is then

$\frac{\mathrm{d} I_{x, z}}{\mathrm{~d} s}=\frac{\partial I_{x, z}}{\partial z}+\frac{\partial I_{x, z}}{\partial x} \frac{\mathrm{d} x}{\mathrm{~d} s}=\frac{\partial I_{x, z}}{\partial z}-P \frac{\partial I_{x, z}}{\partial x}=\eta-\kappa I_{x, z}(4)$

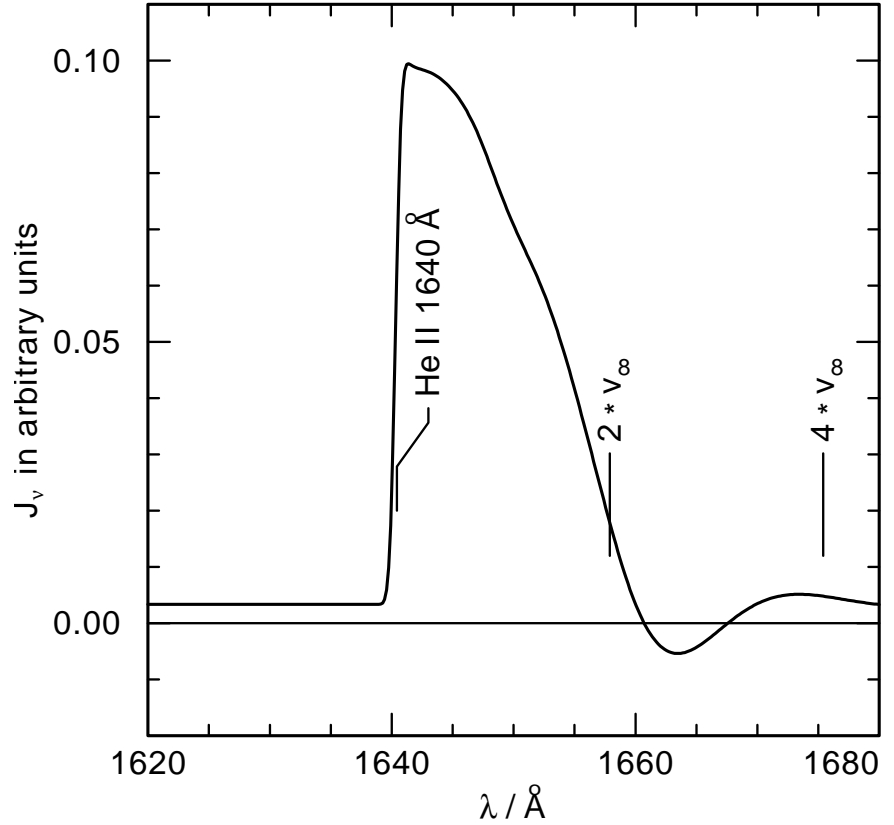

Fig. 1. Example of the mean intensity $J_{\nu}$ at a given depth calculated from a differencing scheme. It shows the outer part of the atmosphere (i.e. the velocity field has almost reached its final value) where the line opacities are low. The terminal velocity is $1600 \mathrm{~km} \mathrm{~s}^{-1}$ with $v_{\text {Dop }}$ being $100 \mathrm{~km} \mathrm{~s}^{-1}$. This leads to a frequency spacing of roughly $0.164 \AA$. Negative intensities occur at wavelengths which correspond to twice the terminal velocity. Redwards of that point the solution oscillates around the continuum value. It takes roughly another $2 \cdot v_{\infty}$ until it is settled.

with the characteristics $P$ defined as

$-P=\frac{\mathrm{d} x}{\mathrm{~d} s}=-\frac{\mu^{2}}{v_{\text {Dop }}} \frac{\mathrm{d} v(r)}{\mathrm{d} r}-\frac{1-\mu^{2}}{r} \frac{v(r)}{v_{\text {Dop }}}$.

The usual way to solve Eq. (4) is to introduce the Feautrier variables $u$ and $v$ which leads, after a discretization in depth and frequency, to a 2nd order difference equation in depth (Mihalas 1978). The frequency derivatives are accounted for in a fully implicit manner.

With respect to the fact that the Eddington factors, calculated from the solution of the ray-by-ray radiation transfer, serve as input to the moment equations, this standard method has one severe disadvantage. In situations where strong lines are calculated together with weak continua, negative mean intensities $J_{\nu}$ or $K_{\nu}$ and therefore negative or even singular Eddington factors $f_{\nu}=K_{\nu} / J_{\nu}$ frequently occur redwards of the line. - Negative $J$ 's do not inevitably enforce negative $K$ 's and vice versa although they are calculated from the same $I$. - The reason is due to numerical overshoot when the radiation field drops from the high value in the line down to a low continuum level. This behavior is illustrated in Fig. 1. Note that frequency derivatives of higher order (e.g. CrankNicholson schemes) are expected to increase the number of negative intensities since they tend to produce numerically unstable results. 


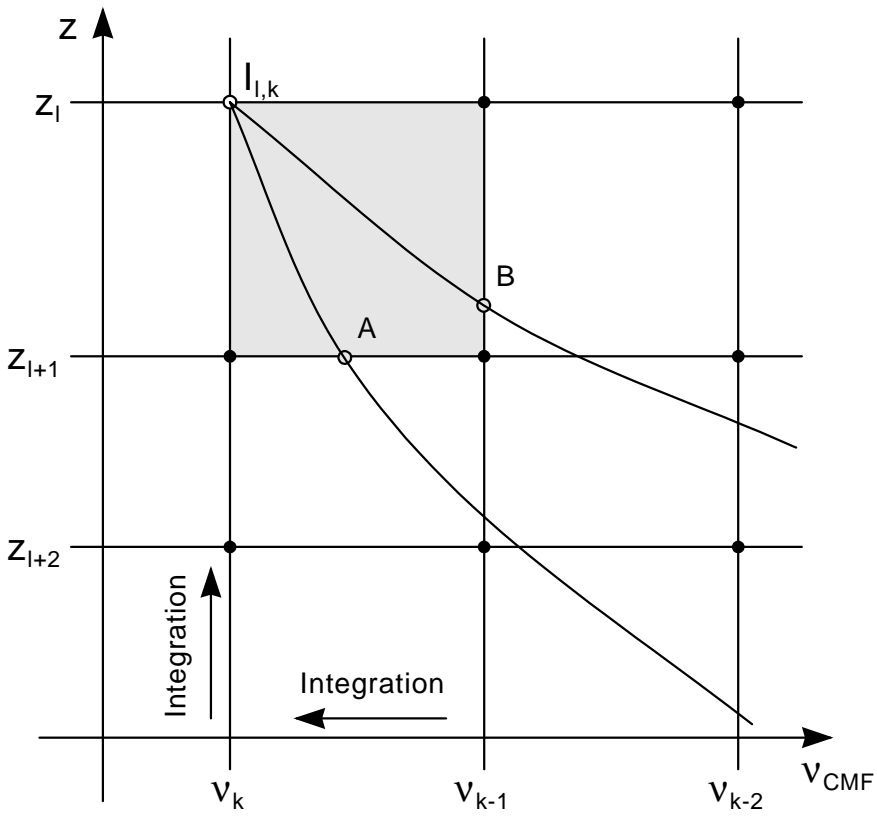

Fig. 2. Integration scheme of the short characteristics method. Known and unknown intensities are denoted by filled and open circles, respectively. The direction of integration is marked by arrows. The curved characteristics intersect the mesh at constant $z$ (case A) or at constant $\nu$ (case $\mathrm{B}$ ). The source functions, opacities and intensities are either interpolated linearly or by cubic splines, respectively.

In our test calculation the terminal velocity is set to $1600 \mathrm{~km} \mathrm{~s}^{-1}$ with $v_{\text {Dop }}$ being $100 \mathrm{~km} \mathrm{~s}^{-1}$. The frequency spacing is set to $0.3 v_{\text {Dop. }}$ The Thomson emissivity is calculated from the continuum radiation field in order to minimize the influence of the preceding solutions.

Negative Eddington factors $f_{\nu}$ have to be replaced by values between 0 and 1 to keep the moment Eqs. (2) and (3) applicable. Unfortunately it turns out that the replacement either by specific values as $0,1 / 3$ or 1 or by any arbitrarily chosen value does not lead to fully satisfying results. Negative Eddington factors slightly change their position in space and frequency from cycle to cycle and cause considerable fluctuations of the occupation numbers. These fluctuations themselves in turn induce changes in the positions. Practically, some model calculations never converge due to this behavior.

In principal it should also be possible to avoid negative intensities by accounting for more depth points (ND). This would be time consuming since the number of operations scale with $\mathrm{ND}^{2}$. Moreover it might be difficult to predict a sufficient radial spacing at the time when the model is started.

In order to overcome these numerical problems, we have replaced the differencing scheme by a direct integration along short characteristics. Figure 2 shows the plane spanned by the spatial coordinate $z$ along a specific ray and the frequency $\nu$.

The integration is performed from blue to red with $\nu_{k}<\nu_{k-1}$. Along a ray at a given frequency the intensity $I_{\nu}$ is at first integrated from the outer boundary to the inner one and at second in the reverse direction. A reflecting inner boundary condition (i.e. $I^{+}=I^{-}$) applies for rays which do not meet the core while a diffusion approximation is applied to the core-rays. To account for the spherical expansion of the atmosphere all quantities as opacities, emissivities and radiation fields are calculated in the CMF. As in the standard Feautrier scheme, the frequency derivatives are also treated in a fully implicit way.

As shown in Fig. 2 we follow the characteristics back to the nearest point of intersection with the grid. To keep the integration simple we introduce two approximations. a: the characteristics in each cell are approximated by a straight line with the slope of the characteristic at the mesh point under consideration. b: the physical quantities as source function, opacity and intensity in the grid are assumed to be linear functions along the characteristics.

Since the points of intersection generally do not coincide with the mesh, auxiliary points have to be introduced. This is done by interpolation either in the frequency or in the spatial coordinate. One advantage of our approach is that only one extra point per integration step is needed. Cubic splines turned out to be essential for the interpolation in the spatial coordinate (case B) while in the frequency coordinate, linear interpolation is sufficient (case A). This reflects the fact that the spatial resolution of the grid is much coarser than the frequency resolution. In case $\mathrm{B}$ the interpolation is performed in $z$, the spatial coordinate, along a ray. It is worth mentioning that these interpolations are necessary due to the expansion of the atmosphere. In a static situation the characteristics would degrade to straight lines directed to the last depth point. This would be a special case A (i.e. without interpolation). As the atmosphere is now assumed to expand, the characteristic curve and the point of intersection moves towards the last frequency. Since the frequency spacing is relatively fine (i.e. $0.3 \cdot v_{\text {Dop }}$ ), errors should be small as long as we encounter case A because the physical quantities change on the $v_{\text {Dop }}$ scale. This justifies a linear approximation. The situation changes substantially for higher expansion rates. The characteristics become shallower and the point of intersection with the underlying depth plane moves without restraint in frequency more and more towards the blue. In principle, the characteristic could cover any frequency range if the expansion rate is high enough. This means that the physical quantities could vary by large amounts along the ray. In that case we need some additional information that is gained at the point where the characteristic intersects the last frequency plane (case B). In contrast to case $\mathrm{A}$ the interpolation has to be performed more carefully because the variation of the physical quantities from depth to depth could be high. Consequently we apply cubic splines.

Two additional notes are needed. (i) It turned out that the profit of the cubic interpolation in case B comes predominantly from the improved intensity rather than from the improved opacity and source function. Therefore the latter quantities could be derived from a linear interpolation. (ii) Case B occurs much more frequently than case 
A since the frequency spacing is much finer than the radial spacing. Only at the spatial boundaries, where the depth points are clustered and where the velocity gradient is small, and/or when coarse frequency steps are applied (i.e. in spectral regions without lines), some cases of type A occur. Therefore errors induced by linear interpolation should be small.

In the following, interpolated values are marked by a circonflex. The outward directed intensity $I^{+}$at a given depth $z_{l}$ and a given frequency $\nu_{k}$ is described by

$I_{l, k}^{+}=\int_{\tau=0}^{\tau} S(\tau) e^{-\tau} \mathrm{d} \tau+\hat{I}^{+} e^{-\tau}$.

$\tau$ denotes the optical depth along the characteristics inside the grid cell under consideration. The integral describes the radiation transfer across the grid cell while the second term is the intensity which enters the grid cell attenuated by the opacity inside the cell. Replacing the integral by a sum leads to

$I_{l, k}^{+}=w_{0} S_{1, \mathrm{k}}+w_{1} \hat{S}+\hat{I}^{+} e^{-\tau}$,

with $w_{0}$ and $w_{1}$ being the integration weights: $w_{0}=1+$ $\left(e^{-\tau}-1\right) / \tau$ and $w_{1}=-e^{-\tau}-\left(e^{-\tau}-1\right) / \tau . S$ is here assumed to be a linear function. Note that the integration of the inward intensity $I^{-}$is principally similar but with a reversed $z$-direction. The whole formalism is described by Olson \& Kunasz (1987) in great detail.

The method of short characteristics has also been applied by Hauschildt (1992) but with some major differences. (i) He always follows the characteristics back to the last depth point, i.e. he never encounters case B. (ii) $\mathrm{He}$ does not approximate the characteristics by a straight line but follow them exactly. (iii) He describes the source function along the ray by parabolic polynoms. The last point might be most important since parabolic interpolation could lead to negative source functions and negative intensities, in principle. This would nullify our major goal, which is to avoid negative Eddington factors. Moreover, as Hauschildt already noted, parabolic interpolation could lead to a numerically unstable scheme.

In the framework of solving the transfer equation in relativistic flows a similar approach has also been applied by Mihalas (1980). He integrates Eq. (4) over the entire length of each ray (i.e. from surface to surface) by assuming appropriate analytical representations of the source function. Appropriate means here that problems due to numerical parasites are minimized.

From Eq. (5) it becomes clear that in our approach all intensities $I_{\nu}$ and therefore also their first and third moments $J_{\nu}$ and $K_{\nu}$ are always positive. As a consequence no negative Eddington factors $f_{\nu}$ can occur. In the case of positive opacities only positive quantities are multiplied and added up in Eq. (5). Even in case of negative opacities (stimulated emission exceeds absorption) the integral remains positive because negative source functions meet negative $\tau$-steps. However, in integration intervals where the opacity changes its sign, the code branches to an integration of $\eta \mathrm{d} z$ instead of $S \mathrm{~d} \tau$ (cf. Eq. (5)).
In Fig. 3 the resulting mean intensities $J$ are plotted for the same example as in Fig. 1. As mentioned above, negative intensities can never occur if the short-characteristics method is applied. In the scattering zone of the line the intensities of both methods differ by a few percent. This is, in principle, unsatisfying but explainable since very different methods have been applied. For both methods the width of the artificial line wing redwards of $2 \cdot v_{\infty}$ is the same. It takes roughly another line width till the intensity settles to the continuum value. This can be understood in our approach at least if case B is considered. The characteristic is repeatedly smeared out by each interpolation to adjacent depth points. As this is only stopped by the spatial boundary conditions, information from the whole velocity space of the atmosphere (i.e. $2 \cdot v_{\infty}$ ) contributes, in principle. Of course, the smearing is attenuated due to the accumulated multiplication by $e^{-\tau}$.

It should be mentioned that even in the case of vanishing opacities the propagation of the blurred information is damped by the interpolation itself. This can be illustrated if we ask for the fraction of intensity transported through frequency space at a fixed depth. - The other portion will vanish via the spatial boundary conditions at least after ND interpolations (ND: number of depth points). - This fraction is $q$ times the intensity itself with $q$ being the interpolation weight (i.e. $q<1$ ). Therefore the propagation of intensity across the frequency space at a fixed depth is damped repeatedly by $q$, with $q$ being 0.5 , typically. This leads in our example, with 53.3 frequency steps per $2 \cdot v_{\infty}$, to a total damping across a line width of about $10^{-16}$.

The dependence of the accuracy on the frequency and depth spacing has been studied by calculating two models with a doubled number of frequency and depth points, respectively. The finer frequency grid leads to slightly stronger artificial red line wing beyond $2 \cdot v_{\infty}$. This can be attributed to an additional blurring of the characteristics from the doubled number of interpolations. In contrast the doubled number of depth points increases the accuracy of both the differencing scheme and the integration along short characteristics (cf. Fig. 3). However, the principle problem of the differencing scheme persists, i.e. the occurrence of negative intensities.

\section{Summary}

We present our scheme for solving the equations of radiation transfer in the co-moving frame. As already suggested by HM the mean intensity $J$ is derived by solving the moment equations in turn with the angle-dependent transfer equation. Usually the latter is solved numerically by applying a differencing scheme. However, the obtained $J$ can suffer from spurious negative values leading to unphysical or singular Eddington factors. To overcome these problems we apply a method of direct integration along short characteristics. It should also be possible to extend the method to the solution of the radiation transfer including relativistic terms. 


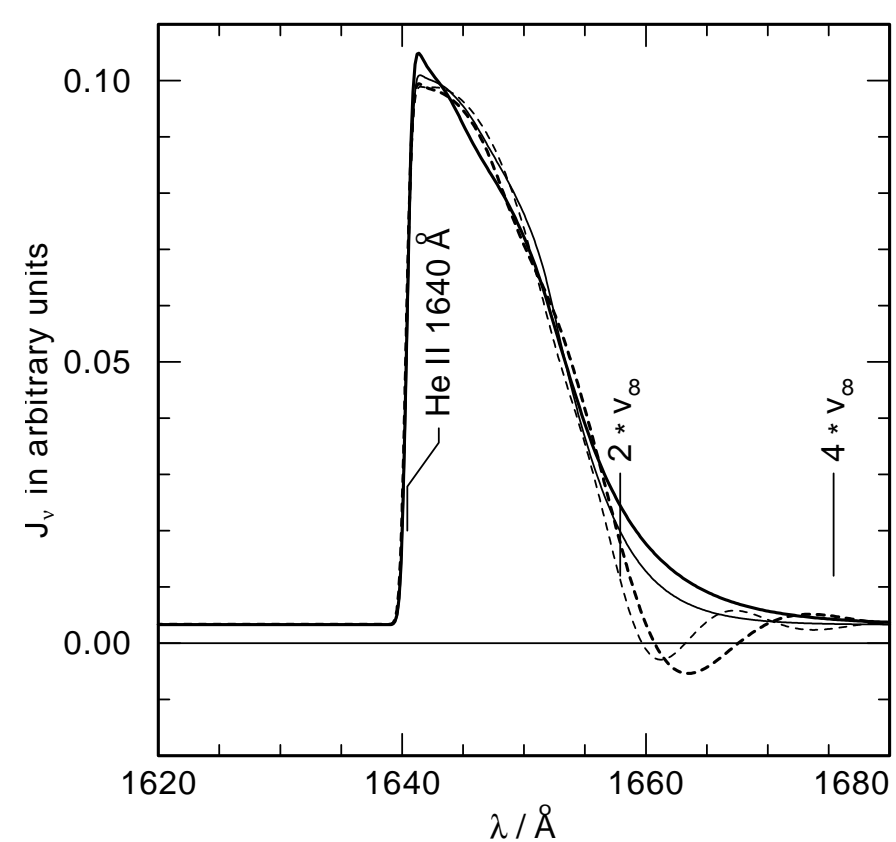

Fig. 3. Mean intensities $J_{\nu}$ from a differencing scheme (thick dashed) and the short characteristics scheme (thick solid). Negative intensities are avoided because the oscillating behavior of the differencing scheme is turned by the shortcharacteristics method into a smooth decline redwards 2 times $v_{\infty}$. The solution of both methods settles down to the continuum value at about $4 \cdot v_{\infty}$. The intensities near the line center (i.e. the scattering zone) differ by about a few percent. Additionally a second pair of curves is shown from a calculation with a doubled number of depth points (thin dashed: differencing scheme; thin solid: short characteristics).

The whole scheme turned out to form a powerful basis for the calculation of spherically expanding non-LTE models. For a realistic model accounting for about 200 non-LTE levels with about
100000 frequency points, the solution of the radiation transfer including a ray-by-ray calculation lasts about 5 min on a modern workstation (e.g. Compaq XP 1000). About half the time is spent on the calculation of the opacities while the other half is spent on the ray-by-ray solution. In terms of CPU time the differencing and the integral method turned out to be equivalent. About $100 \mathrm{MB}$ disk space are required to store the Eddington factors.

In a subsequent paper (Koesterke in prep.) we will describe how the equations of the statistical equilibrium are solved consistently. The construction of the lambda operator from the short characteristics scheme will also be treated. This will be the starting point for our investigation of the atmospheres of massive Wolf-Rayet stars and central stars of planetary nebulae of Wolf-Rayet type.

\section{References}

Anderson, L. S. 1989, ApJ, 339, 558

Auer, L. H., \& Mihalas, D. 1970, MNRAS, 149, 65

Dreizler, S., \& Werner, K. 1993, A\&A, 278, 199

Gräfener, G., Koesterke, L., \& Hamann, W.-R. 2001, A\&A, in preparation

Hamann, W.-R. 1987, in Numerical Radiative Transfer, ed. W. Kalkofen (Cambridge University Press), 35

Hauschildt, P. H. 1992, JQSRT, 47, 433

Hillier, D. J., \& Miller, D. L. 1998, ApJ, 496, 407

Hubeny, I., \& Lanz, T. 1995, ApJ, 439, 875

Koesterke, L. 2001, A\&A, in preparation

Mihalas, D. 1978, Stellar Atmospheres, 2nd ed. (San Francisco: Freeman)

Mihalas, D. 1980, ApJ, 237, 574

Mihalas, D., Kunasz, P. B., \& Hummer, D. G. 1976, ApJ, 206, 515

Olson, G. L., \& Kunasz, P. B. 1987, JQSRT, 38, 325 